\title{
DIRICHLET SPACES ASSOCIATED WITH LOCALLY FINITE ROOTED DIRECTED TREES
}

\author{
SAMEER CHAVAN, DEEPAK KUMAR PRADHAN, AND SHAILESH TRIVEDI
}

\begin{abstract}
Let $\mathscr{T}=(V, \mathcal{E})$ be a leafless, locally finite rooted directed tree. We associate with $\mathscr{T}$ a one parameter family of Dirichlet spaces $\mathscr{H}_{q}(q \geqslant 1)$, which turn out to be Hilbert spaces of vector-valued holomorphic functions defined on the unit disc $\mathbb{D}$ in the complex plane. These spaces can be realized as reproducing kernel Hilbert spaces associated with the positive definite kernel

$$
\begin{aligned}
\kappa_{\mathscr{H}_{q}}(z, w) & =\sum_{n=0}^{\infty} \frac{(1)_{n}}{(q)_{n}} z^{n} \bar{w}^{n} P_{\left\langle e_{\text {root }}\right\rangle} \\
& +\sum_{v \in V \prec} \sum_{n=0}^{\infty} \frac{\left(n_{v}+2\right)_{n}}{\left(n_{v}+q+1\right)_{n}} z^{n} \bar{w}^{n} P_{v}(z, w \in \mathbb{D}),
\end{aligned}
$$

where $V_{\prec}$ denotes the set of branching vertices of $\mathscr{T}, n_{v}$ denotes the depth of $v \in V$ in $\mathscr{T}$, and $P_{\left\langle e_{\text {root }}\right\rangle}, P_{v}\left(v \in V_{\prec}\right)$ are certain orthogonal projections. Further, we discuss the question of unitary equivalence of operators $\mathscr{M}_{z}^{(1)}$ and $\mathscr{M}_{z}^{(2)}$ of multiplication by $z$ on Dirichlet spaces $\mathscr{H}_{q}$ associated with directed trees $\mathscr{T}_{1}$ and $\mathscr{T}_{2}$ respectively.
\end{abstract}

\section{Introduction}

The present work is a sequel to [17. In that paper, a rich interplay between the directed trees and analytic kernels of finite bandwidth has been exploited to study the weighted shifts on directed trees. The analysis therein was based on Shimorin's analytic model as introduced in [28] (for an alternate approach to the function theory of weighted shifts on directed trees, the reader is referred to [14, [13]). Our work is also motivated partly by the classification theorem [6, Theorem 9.9] obtained for 2-isometric weighted shifts on certain directed trees.

The objective of the present paper is to introduce Dirichlet spaces associated with certain rooted directed trees. This is carried out by introducing the so-called Dirichlet shifts on directed trees with weights being certain functions of depth of vertices, and thereafter applying Shimorin's construction [28] to these shifts. These spaces can be thought of as vector-valued weighted Dirichlet spaces (cf. 25]). We also discuss the spaces Cauchy dual to Dirichlet spaces. These turn out to be vectorvalued Bergman spaces, which play a key role in answering the question of unitary equivalence of Dirichlet shifts associated with two directed trees. We collect below some preliminaries required to define the Dirichlet shifts. For a detailed exposition on weighted shifts on directed trees, the reader is referred to [23] and [17].

A directed graph is a pair $\mathscr{T}=(V, \mathcal{E})$, where $V$ is a nonempty set and $\mathcal{E}$ is a nonempty subset of $V \times V \backslash\{(v, v): v \in V\}$. An element of $V$ (resp. $\mathcal{E}$ ) is called a vertex (resp. an edge) of $\mathscr{T}$. A finite sequence $\left\{v_{i}\right\}_{i=1}^{n}$ of distinct vertices is said to be a circuit in $\mathscr{T}$ if $n \geqslant 2,\left(v_{i}, v_{i+1}\right) \in \mathcal{E}$ for all $1 \leqslant i \leqslant n-1$ and $\left(v_{n}, v_{1}\right) \in \mathcal{E}$. We say that two distinct vertices $u$ and $v$ of $\mathscr{T}$ are connected by a path if there exists

2010 Mathematics Subject Classification. Primary 46E22, 31C25; Secondary 47B20, 05C20.

Key words and phrases. Dirichlet space, directed tree, $q$-isometry.

The work of the second author is supported through the NBHM Research Fellowship. 
a finite sequence $\left\{v_{i}\right\}_{i=1}^{n}$ of distinct vertices of $\mathscr{T}(n \geqslant 2)$ such that $v_{1}=u, v_{n}=v$ and $\left(v_{i}, v_{i+1}\right)$ or $\left(v_{i+1}, v_{i}\right) \in \mathcal{E}$ for all $1 \leqslant i \leqslant n-1$. A directed graph $\mathscr{T}$ is said to be connected if any two distinct vertices of $\mathscr{T}$ can be connected by a path in $\mathscr{T}$. For a subset $W$ of $V$, define

$$
\operatorname{Chi}(W):=\bigcup_{u \in W}\{v \in V:(u, v) \in \mathcal{E}\} .
$$

We define inductively $\mathrm{Chi}^{\langle n\rangle}(W)$ for $n \in \mathbb{N}$ as follows:

$$
\mathrm{Chi}^{\langle n\rangle}(W):= \begin{cases}W & \text { if } n=0, \\ \operatorname{Chi}\left(\mathrm{Chi}^{\langle n-1\rangle}(W)\right) & \text { if } n \geqslant 1 .\end{cases}
$$

Given $v \in V$, we write $\operatorname{Chi}(v):=\operatorname{Chi}(\{v\})$. An element of $\operatorname{Chi}(v)$ is called a child of $v$. For a given vertex $v \in V$, consider the set $\operatorname{Par}(v):=\{u \in V:(u, v) \in \mathcal{E}\}$. If $\operatorname{Par}(v)$ is singleton, then the unique vertex in $\operatorname{Par}(v)$ is called the parent of $v$, which we denote by $\operatorname{par}(v)$. Let the $\operatorname{subset} \operatorname{Root}(\mathscr{T})$ of $V$ be defined as

$$
\operatorname{Root}(\mathscr{T}):=\{v \in V: \operatorname{Par}(v)=\emptyset\}
$$

Then an element of $\operatorname{Root}(\mathscr{T})$ is called a root of $\mathscr{T}$. If $\operatorname{Root}(\mathscr{T})$ is $\operatorname{singleton}$, then its unique element is denoted by root. We set $V^{\circ}:=V \backslash \operatorname{Root}(\mathscr{T})$. A directed graph $\mathscr{T}=(V, \mathcal{E})$ is called a directed tree if $\mathscr{T}$ has no circuits, $\mathscr{T}$ is connected and each vertex $v \in V^{\circ}$ has a unique parent. A directed tree $\mathscr{T}$ is said to be

(i) rooted if it has a unique root.

(ii) locally finite if card $(\operatorname{Chi}(u))$ is finite for all $u \in V$, where card $(X)$ stands for the cardinality of the set $X$.

(iii) leafless if every vertex has at least one child.

Let $\mathscr{T}=(V, \mathcal{E})$ be a rooted directed tree with root root. Let $V_{\prec}$ be the set $\{u \in V: \operatorname{card}(\operatorname{Chi}(u)) \geqslant 2\}$ of branching vertices of $\mathscr{T}$. For each $u \in V$, the depth of $u$ is the unique non-negative integer $n_{u}$ such that $u \in \mathrm{Chi}^{\left\langle n_{u}\right\rangle}$ (root). Define the branching index of $\mathscr{T}$ as

$$
k_{\mathscr{T}}:= \begin{cases}1+\sup \left\{n_{w}: w \in V_{\prec}\right\} & \text { if } V_{\prec} \text { is non-empty, } \\ 0 & \text { if } V_{\prec} \text { is empty. }\end{cases}
$$

We say that $\mathscr{T}$ is of finite branching index if $k_{\mathscr{T}}<\infty$. We further say that two directed trees are isomorphic if there exists a bijection between their sets of vertices which preserves directed edges.

Let $\mathscr{T}=(V, \mathcal{E})$ be a rooted directed tree with root root. We always assume that $\operatorname{card}(V)=\aleph_{0}$. In what follows, $l^{2}(V)$ stands for the Hilbert space of square summable complex functions on $V$ equipped with the standard inner product. Note that the set $\left\{e_{u}\right\}_{u \in V}$ is an orthonormal basis of $l^{2}(V)$, where $e_{u} \in l^{2}(V)$ is the indicator function of $\{u\}$. Given a system $\boldsymbol{\lambda}=\left\{\lambda_{v}\right\}_{v \in V^{\circ}}$ of non-negative real numbers, we define the weighted shift operator $S_{\lambda}$ on $\mathscr{T}$ with weights $\boldsymbol{\lambda}$ by

$$
\begin{aligned}
\mathscr{D}\left(S_{\lambda}\right) & :=\left\{f \in l^{2}(V): \Lambda_{\mathscr{T}} f \in l^{2}(V)\right\}, \\
S_{\lambda} f & :=\Lambda_{\mathscr{T}} f, \quad f \in \mathscr{D}\left(S_{\lambda}\right),
\end{aligned}
$$

where $\Lambda_{\mathscr{T}}$ is the mapping defined on complex functions $f$ on $V$ by

$$
\left(\Lambda_{\mathscr{T}} f\right)(v):= \begin{cases}\lambda_{v} \cdot f(\operatorname{par}(v)) & \text { if } v \in V^{\circ}, \\ 0 & \text { if } v \text { is a root of } \mathscr{T} .\end{cases}
$$

Remark 1.1. If $S_{\lambda}$ is bounded then $S_{\lambda}^{*} e_{u}=\lambda_{u} e_{\operatorname{par}(u)}$ if $u \neq \operatorname{root}$, and $S_{\lambda}^{*} e_{u}=0$ otherwise. 
Throughout these paper, we will be interested in weighted shifts which are bounded linear. The reader is referred to [23] for the basic theory of weighted shifts on directed trees. In particular, it may be concluded from [23, Proposition 3.5.1(ii)] that for a rooted directed tree $\mathscr{T}$ with root root, the kernel of $S_{\lambda}^{*}$ is given by

$$
E=\left\langle e_{\text {root }}\right\rangle \oplus \bigoplus_{v \in V_{\prec}}\left(l^{2}(\operatorname{Chi}(v)) \ominus\left\langle\boldsymbol{\lambda}^{v}\right\rangle\right)
$$

where $\boldsymbol{\lambda}^{v}:$ Chi $(v) \rightarrow \mathbb{C}$ is defined by $\boldsymbol{\lambda}^{v}(u)=\lambda_{u}$ and $\langle f\rangle$ denotes the span of $\{f\}$.

Recall that a bounded linear operator $T$ on a Hilbert space $\mathcal{H}$ is finitely multicyclic if there are a finite number of vectors $h_{1}, \cdots, h_{m}$ in $\mathcal{H}$ such that

$$
\mathcal{H}=\bigvee\left\{T^{k} h_{1}, \cdots, T^{k} h_{m}: k \in \mathbb{N}\right\}
$$

Remark 1.2. Let $\mathscr{T}$ be a leafless, locally finite rooted directed tree with finite branching index and let $S_{\lambda}$ be a bounded weighted shift on $\mathscr{T}$. It may be concluded from [17, Proposition 2.1 and Corollary 2.3] that $S_{\lambda}$ is finitely cyclic.

\section{Dirichlet Shifts on Directed Trees}

We now introduce the notion of Dirichlet shift on certain directed trees.

Definition 2.1. Let $\mathscr{T}=(V, \mathcal{E})$ be a leafless, locally finite rooted directed tree. For a real number $q \geqslant 1$, consider the weighted shift $S_{\lambda, q}$ on $\mathscr{T}$ with weights given by

$$
\lambda_{u, q}=\frac{1}{\sqrt{\operatorname{card}(\operatorname{Chi}(v))}} \sqrt{\frac{n_{v}+q}{n_{v}+1}} \quad \text { for } u \in \operatorname{Chi}(v), v \in V,
$$

where $n_{v}$ is the depth of $v \in V$ in $\mathscr{T}$. We refer to $S_{\lambda, q}$ as the Dirichlet shift on $\mathscr{T}$.

Let $S_{\lambda, q}$ be a Dirichlet shift on $\mathscr{T}$. Note that the weights of $S_{\lambda, q}$ can be rewritten as

$$
\lambda_{v, q}=\sqrt{\frac{n_{v}+q-1}{n_{v}}} \frac{1}{\sqrt{\operatorname{card}(\operatorname{sib}(v))}} \text { for } v \in V^{\circ},
$$

where, for $u \in V$, the siblings of $u$ is given by

$$
\operatorname{sib}(u):= \begin{cases}\text { Chi }(\operatorname{par}(u)) & \text { if } u \neq \text { root } \\ \emptyset & \text { otherwise }\end{cases}
$$

Note further that

$$
\sup _{v \in V} \sum_{u \in \operatorname{Chi}(v)} \lambda_{u, q}^{2}=\sup _{v \in V} \frac{n_{v}+q}{n_{v}+1}=q, \quad \inf _{v \in V} \sum_{u \in \operatorname{Chi}(v)} \lambda_{u, q}^{2}=\inf _{v \in V} \frac{n_{v}+q}{n_{v}+1}=1 .
$$

It now follows from [23. Propositions 3.1.8 and 3.6.1] that $S_{\lambda, q}$ is bounded linear, and left-invertible (that is, $S_{\lambda, q}$ is one-one with closed range). Thus the Cauchy dual $S_{\lambda, q}^{\prime}$ of $S_{\lambda, q}$ given by $S_{\lambda, q}\left(S_{\lambda, q}^{*} S_{\lambda, q}\right)^{-1}$ is well-defined [28]. It turns out that $S_{\lambda, q}^{\prime}$ is a weighted shift $S_{\lambda^{\prime}, q}$ on $\mathscr{T}$ with weights given by

$$
\lambda_{v, q}^{\prime}=\sqrt{\frac{n_{v}}{n_{v}+q-1}} \frac{1}{\sqrt{\operatorname{card}(\operatorname{sib}(v))}} \text { for all } v \in V^{\circ} .
$$

Remark 2.2. In case $\mathscr{T}$ is a rooted directed tree without any branching vertex (that is, $\mathscr{T}$ is isomorphic to $\mathbb{N}), S_{\lambda, q}$ is unitarily equivalent to the classical weighted shift $S_{w, q}$ with weights $w:=\left\{\sqrt{\frac{n+q}{n+1}}\right\}_{n \in \mathbb{N}}$ (classical Dirichlet shift). Note that the weights of the Cauchy dual $S_{w, q}^{\prime}$ of $S_{w, q}$ are $\left\{\sqrt{\frac{n+1}{n+q}}\right\}_{n \in \mathbb{N}}$ (classical Bergman shift). 
The following classification problem is motivated by [6, Theorem 9.9]:

Problem 2.3. Let $S_{\lambda, q}^{(1)}, S_{\lambda, q}^{(2)}$ be Dirichlet shifts on $\mathscr{T}_{1}, \mathscr{T}_{2}$ respectively. Find sufficient and necessary conditions on $\mathscr{T}_{1}$ and $\mathscr{T}_{2}$ which ensure that $S_{\lambda, q}^{(1)}$ and $S_{\lambda, q}^{(2)}$ are unitarily equivalent.

Note that the above problem has the following solution in case $q=1$. The shifts $S_{\lambda, 1}^{(1)}$ and $S_{\lambda, 1}^{(2)}$ unitarily equivalent if and only if

$$
\sum_{v \in V_{\prec}^{(1)}}(\operatorname{card}(\operatorname{Chi}(v))-1)=\sum_{v \in V_{\prec}^{(2)}}(\operatorname{card}(\operatorname{Chi}(v))-1),
$$

where $V_{\prec}^{(1)}$ and $V_{\prec}^{(2)}$ are set of branching vertices of $\mathscr{T}_{1}$ and $\mathscr{T}_{2}$ respectively. Indeed, since $S_{\lambda, 1}^{(1)}$ and $S_{\lambda, 1}^{(2)}$ are isometries (Proposition 2.7 below), by von Neumann-Wold decomposition [18, $S_{\lambda, 1}^{(1)}$ and $S_{\lambda, 1}^{(2)}$ unitarily equivalent if and only if $\operatorname{dim} \operatorname{ker}\left(S_{\lambda, 2}^{(1)}\right)^{*}=$ $\operatorname{dim} \operatorname{ker}\left(S_{\lambda, 2}^{(2)}\right)^{*}$. The desired conclusion is now immediate from (1.1). This has been recorded in more generality in [6, Theorem 9.9(ii)].

One of the main results of this note provides a solution to the above problem in case $q$ is a positive integer.

Theorem 2.4. Let $q$ be an integer bigger than 1 . For $j=1,2$, let $S_{\lambda, q}^{(j)}$ be the Dirichlet shift on rooted directed tree $\mathscr{T}_{j}=\left(V_{j}, \mathcal{E}_{j}\right)$ with $\operatorname{root}_{j}$, let $\mathcal{G}_{n}^{(j)}:=\left\{v \in V_{j}\right.$ : $\left.v \in \mathrm{Chi}^{\langle n\rangle}\left(\operatorname{root}_{j}\right)\right\}(n \in \mathbb{N})$, and $E_{j}=\operatorname{ker}\left(S_{\lambda, q}^{(j)}\right)^{*}$. Then $S_{\lambda, q}^{(1)}$ is unitarily equivalent to $S_{\lambda, q}^{(2)}$ if and only if for every $n \in \mathbb{N}$,

$$
\sum_{v \in V_{\prec}^{(1)} \cap \mathcal{G}_{n}^{(1)}}(\operatorname{card}(\operatorname{Chi}(v))-1)=\sum_{v \in V_{\prec}^{(1)} \cap \mathcal{G}_{n}^{(2)}}(\operatorname{card}(\operatorname{Chi}(v))-1) .
$$

Remark 2.5. Since $\mathscr{T}_{j}$ is locally finite, the $V_{\prec}^{(j)} \cap \mathcal{G}_{n}^{(j)}$ is finite for every $n \in \mathbb{N}$ and $j=1,2$, and hence the sums appearing in (2.3) are finite. Further, it may happen that (2.3) holds for two non-isomorphic directed trees (see [6, Figure 2]).

The case $q=2$ of Theorem 2.4 is a special case of [6, Theorem 9.9(i)]. In what follows, we provide an alternative verification of this fact based on modeling $S_{\lambda, 2}$ as a multiplication by $z$ on a vector-valued Dirichlet space. With this identification, the problem essentially reduces to classification problem of multiplication operators on vector-valued Dirichlet spaces (refer to Section 3). This part of the proof relies on the theory of vector-valued Dirichlet spaces as expounded in [25]. The rather involved proof of the general case, as presented in the last section, relies on tree analogs of weighted Bergman spaces. These spaces can be seen as Cauchy dual of Dirichlet spaces in the sense of S. Shimorin [28].

In the remaining part of this section, we derive some structural properties of the weighted shifts $S_{\lambda, q}$ on $\mathscr{T}$. Before we state formulae for moments of $S_{\lambda, q}$ and $S_{\lambda^{\prime}, q}$, recall that the Pochhammer symbol is defined by

$$
(x)_{y}=\frac{\Gamma(x+y)}{\Gamma(x)}
$$

where $\Gamma$ is the gamma function defined for all complex numbers except the nonpositive integers.

Lemma 2.6. Let $S_{\lambda, q}$ be a Dirichlet shift on $\mathscr{T}=(V, \mathcal{E})$ and let $S_{\lambda^{\prime}, q}$ be the Cauchy dual of $S_{\lambda, q}$. Then for $k \in \mathbb{N}$ and $v \in V$,

$$
\left\|S_{\lambda, q}^{k} e_{v}\right\|^{2}=\frac{\left(n_{v}+q\right)_{k}}{\left(n_{v}+1\right)_{k}}, \quad\left\|S_{\lambda^{\prime}, q}^{k} e_{v}\right\|^{2}=\frac{\left(n_{v}+1\right)_{k}}{\left(n_{v}+q\right)_{k}} .
$$


In particular, the spectral radii of $S_{\lambda, q}$ and $S_{\lambda^{\prime}, q}$ are 1.

Proof. We verify the first formula by induction on integers $k \geqslant 0$ for a fixed $v \in V$. The formula is trivial for $k=0$. Suppose the formula holds for some integer $k \geqslant 0$. Since $\mathrm{Chi}^{\langle n\rangle}(u)$ and $\mathrm{Chi}^{\langle n\rangle}(w)$ are disjoint for distinct vertices $u$ and $w$, it follows from [23, Lemma 6.1.1(i)] that $\left\{S_{\lambda, q}^{k} e_{w}\right\}_{w \in \operatorname{Chi}(v)}$ is mutually orthogonal. Also, since $n_{w}=n_{v}+1$ for $w \in \operatorname{Chi}(v)$, we obtain

$$
\begin{aligned}
\left\|S_{\lambda, q}^{k+1} e_{v}\right\|^{2} & =\left\|S_{\lambda, q}^{k} \sum_{w \in \operatorname{Chi}(v)} \lambda_{w, q} e_{w}\right\|^{2}=\sum_{w \in \operatorname{Chi}(v)} \lambda_{w, q}^{2}\left\|S_{\lambda, q}^{k} e_{w}\right\|^{2} \\
& \stackrel{\text { 2.11 }}{=} \sum_{w \in \operatorname{Chi}(v)} \frac{1}{\operatorname{card}(\operatorname{Chi}(v))} \frac{n_{v}+q}{n_{v}+1} \frac{\left(n_{w}+q\right)_{k}}{\left(n_{w}+1\right)_{k}} \\
& =\frac{n_{v}+q}{n_{v}+1} \frac{\left(n_{v}+q+1\right)_{k}}{\left(n_{v}+2\right)_{k}}=\frac{\left(n_{v}+q\right)_{k+1}}{\left(n_{v}+1\right)_{k+1}} .
\end{aligned}
$$

The second formula can be verified similarly. To see the remaining part, note that

$$
\left\|S_{\lambda, q}^{k}\right\|=\sup _{n \in \mathbb{N}} \sqrt{\frac{(n+q)_{k}}{(n+1)_{k}}}, \quad\left\|S_{\lambda^{\prime}, q}^{k}\right\|=\sup _{n \in \mathbb{N}} \sqrt{\frac{(n+1)_{k}}{(n+q)_{k}}}
$$

and apply the spectral radius formula.

The second part of the following generalizes [8, Proposition 8], [3, Theorem 8.6].

Proposition 2.7. Let $S_{\lambda, q}$ be a Dirichlet shift on $\mathscr{T}=(V, \mathcal{E})$ and let $S_{\lambda^{\prime}, q}$ be the Cauchy dual of $S_{\lambda, q}$. If $q$ is a positive integer, then we have the following:

(i) $S_{\lambda^{\prime}, q}$ is subnormal, that is, $S_{\lambda^{\prime}, q}$ admits a normal extension.

(ii) $S_{\lambda, q}$ is a q-isometry, that is,

$$
\sum_{k=0}^{q}(-1)^{k}\left(\begin{array}{l}
q \\
k
\end{array}\right) S_{\lambda, q}^{* k} S_{\lambda, q}^{k}=0
$$

but not a $(q-1)$-isometry,

(iii) if $\mathscr{T}$ is of finite branching index, then the self-commutator $\left[S_{\lambda, q}^{*}, S_{\lambda, q}\right]:=$ $S_{\lambda, q}^{*} S_{\lambda, q}-S_{\lambda, q} S_{\lambda, q}^{*}$ of $S_{\lambda, q}$ is of trace-class,

(iv) $S_{\lambda, 2}$ has wandering subspace property, that is, for any $S_{\lambda, 2}$-invariant subspace $\mathcal{M}$ of $l^{2}(V)$,

$$
\mathcal{M}=\bigvee_{k \in \mathbb{N}}\left\{S_{\lambda, 2}^{k} f: f \in \mathcal{M} \ominus S_{\lambda, 2} \mathcal{M}\right\}
$$

Proof. Suppose that $q$ is a positive integer.

(i) By [23, Theorem 6.1.3], $S_{\lambda^{\prime}, q}$ is subnormal if and only if for every $v \in V$, $\left\{\left\|S_{\lambda^{\prime}, q}^{k} e_{v}\right\|^{2}\right\}_{k \in \mathbb{N}}$ is a Hausdorff moment sequence. However, by Lemma 2.6, for $v \in V$ and $k \in \mathbb{N}$.

$$
\left\|S_{\lambda^{\prime}, q}^{k} e_{v}\right\|^{2}=\frac{\left(n_{v}+1\right)_{k}}{\left(n_{v}+q\right)_{k}}= \begin{cases}1 & \text { if } q=1, \\ \frac{\left(n_{v}+1\right) \cdots\left(n_{v}+q-1\right)}{\left(n_{v}+k+1\right) \cdots\left(n_{v}+k+q-1\right)} & \text { if } q \geqslant 2 .\end{cases}
$$

Since $\left\{\frac{1}{k+l}\right\}_{k \in \mathbb{N}}$ is a Hausdorff moment sequence for any integer $l \geqslant 1$, by general theory [10], so is $\left\{\left\|S_{\lambda^{\prime}, q}^{k} e_{v}\right\|^{2}\right\}_{k \in \mathbb{N}}$.

(ii) Since the sequence $\left\{S_{\lambda, q}^{k} e_{v}\right\}_{v \in V}$ is orthogonal, it is sufficient to check that

$$
\sum_{k=0}^{q}(-1)^{k}\left(\begin{array}{l}
q \\
k
\end{array}\right)\left\|S_{\lambda, q}^{k} e_{v}\right\|^{2}=0 \text { for every } v \in V .
$$


However, by Lemma 2.6, for $v \in V$ and $k \in \mathbb{N}$,

$$
\left\|S_{\lambda, q}^{k} e_{v}\right\|^{2}=\frac{\left(n_{v}+q\right)_{k}}{\left(n_{v}+1\right)_{k}}= \begin{cases}1 & \text { if } q=1 \\ \frac{\left(n_{v}+k+1\right) \cdots\left(n_{v}+k+q-1\right)}{\left(n_{v}+1\right) \cdots\left(n_{v}+q-1\right)} & \text { if } q \geqslant 2 .\end{cases}
$$

In any case, the sequence $\left\{\left\|S_{\lambda, q}^{k} e_{v}\right\|^{2}\right\}_{k \in \mathbb{N}}$ is a polynomial in $k$ of degree $q-1$. By 16. Proof of Lemma 2.5], $S_{\lambda, q}$ is a $q$-isometry, but not a $(q-1)$-isometry.

(iii) Assume that $\mathscr{T}$ is of finite branching index. By Remark 1.2, $S_{\lambda^{\prime}, q}$ is finitely cyclic. By part (ii) above, $S_{\lambda^{\prime}, q}$ is subnormal. Hence, by Berger-Shaw Theorem [18, $S_{\lambda^{\prime}, q}$ has trace-class self-commutator. Since $A:=S_{\lambda, q}$ is right Fredholm with right essential inverse $\left(A^{*} A\right)^{-1} A^{*}$, the desired conclusion may now be derived from the following identity:

$$
\left[A^{*}, A\right] A=-A^{*} A\left(\left[A^{\prime *}, A^{\prime}\right] A\right) A^{*} A,
$$

where $A^{\prime}:=A\left(A^{*} A\right)^{-1}$.

(iv) This is immediate from part (ii), [17, Lemma 3.3] and [26, Theorem 1].

Remark 2.8. Assume that $\mathscr{T}$ is of finite branching index. Since essential spectral picture of a finitely multicyclic, completely non-unitary $q$-isometry $T$ coincides with the unilateral shift of multiplicity $\operatorname{dim} \operatorname{ker} T^{*}[2$, it may be concluded from the BDF Theorem 12 that $S_{\lambda, q}$ is unitarily equivalent to a compact perturbation of the unilateral shift of multiplicity $\operatorname{dim} \operatorname{ker} S_{\lambda, q}^{*}$.

The shift operators $S_{\lambda, q}(q \geqslant 1)$ provide new examples of finitely multicyclic $q$-isometries in the following sense (cf. [22, Remark 4.5]).

Proposition 2.9. Let $S_{\lambda, q}$ be a Dirichlet shift on a directed tree $\mathscr{T}=(V, \mathcal{E})$ of finite branching index and let $S_{w, q}$ be the classical Dirichlet shift (see Remark [2.2). Then $S_{\lambda, q}$ is unitarily equivalent to any finite orthogonal sum of $S_{w, q}$ if and only if either $q=1$ or $\mathscr{T}$ is isomorphic to $\mathbb{N}$.

Proof. Note that $l=\operatorname{dim} \operatorname{ker} S_{\lambda, q}^{*}$ is finite by [17, Proposition 2.1]. If $\mathscr{T}$ is isomorphic to $\mathbb{N}$ then clearly $S_{\lambda, q}$ is unitarily equivalent to $S_{w, q}$. Further, if $q=1$ then by the von Neumann-Wold decomposition for isometries [18], $S_{\lambda, q}$ is unitarily equivalent to orthogonal sum of $\operatorname{dim} \operatorname{ker} S_{\lambda, q}^{*}$ copies of $S_{w, q}$. This gives the sufficiency part. To see the necessity part, suppose that $S_{\lambda, q}$ is unitarily equivalent to orthogonal sum $S_{w, q}^{(l)}$ of $l$ copies of $S_{w, q}$. Note that the Cauchy dual $S_{\lambda^{\prime}, q}$ of $S_{\lambda, q}$ is unitarily equivalent to $\left(S_{w, q}^{\prime}\right)^{(l)}$. By [16, Example 2.7], the defect operator $\mathcal{D}_{S_{w, q}^{\prime}}$ is an orthogonal projection onto $\operatorname{ker} S_{w, q}^{\prime *}$, where, for a bounded linear operator $T$, $\mathcal{D}_{T}$ is given by

$$
\mathcal{D}_{T}:=\sum_{k=0}^{q}(-1)^{k}\left(\begin{array}{l}
q \\
k
\end{array}\right) T^{k} T^{* k}
$$

The essential part of the proof shows that the defect operator $\mathcal{D}_{S_{\lambda^{\prime}, q}}$ is never an orthogonal projection unless $q=1$ or $\mathscr{T}$ is isomorphic to $\mathbb{N}$.

We may assume that $\mathscr{T}$ is not isomorphic to $\mathbb{N}$. Thus $V_{\prec}$ is nonempty. Further, since $\mathscr{T}$ is locally finite with finite branching index, we can choose $v \in V_{\prec}$ such that $\operatorname{Chi}(v)=\left\{u_{1}, u_{2}, \cdots, u_{m}\right\}$ and $\operatorname{card}\left(\operatorname{Chi}\left(u_{j}\right)\right)=1(j=1, \cdots, m)$ for some positive integer $m \geqslant 2$. Let Chi $\left(u_{j}\right)=\left\{w_{j}\right\}(j=1, \cdots, m)$ and $f_{v}=\sum_{j=1}^{m} f\left(w_{j}\right) e_{w_{j}}$ be such that

$$
\sum_{j=1}^{m} f\left(w_{j}\right)=0, \text { and } f\left(w_{j}\right) \neq 0 \text { for some } j=1, \cdots, m
$$


Note that $S_{\lambda^{\prime}, q}^{*}\left(f_{v}\right) \neq 0$ and $S_{\lambda^{\prime}, q}^{* k}\left(f_{v}\right)=0$ for all integers $k>1$. It then follows that

$$
\begin{aligned}
\sum_{k=0}^{q}(-1)^{k}\left(\begin{array}{l}
q \\
k
\end{array}\right) S_{\lambda^{\prime}, q}^{k} S_{\lambda^{\prime}, q}^{* k}\left(f_{v}\right) & =f_{v}-q \sum_{j=1}^{m} f\left(w_{j}\right) \lambda_{w_{j}}^{\prime} e_{w_{j}} \\
& \stackrel{(2.2)}{=} f_{v}-q \sum_{j=1}^{n} f\left(w_{j}\right) \frac{n_{w_{j}}}{n_{w_{j}}+q-1} e_{w_{j}} \\
& =\left(1-q\left(\frac{n_{v}+2}{n_{v}+q+1}\right)\right) f_{v} .
\end{aligned}
$$

It is easy to see from (1.1) that $f_{v}$ is orthogonal to $\operatorname{ker}\left(S_{\lambda^{\prime}, q}^{*}\right)$. Thus if $D_{S_{\lambda^{\prime}, q}}$ is the orthogonal projection onto $\operatorname{ker}\left(S_{\lambda^{\prime}, q}^{*}\right)$ then we must have

$$
\left(1-q\left(\frac{n_{v}+2}{n_{v}+q+1}\right)\right) f_{v}=0,
$$

that is, $(1-q)\left(n_{v}+1\right)=0$. This is possible only if $q=1$.

\section{Dirichlet Spaces Associated with Directed Trees}

The following result enables us to associate a Dirichlet space with every leafless, locally finite rooted directed tree. It is worth mentioning that certain Hardy-type spaces are associated with some infinite acyclic, undirected, connected graphs in 4 (refer also to [7, Section 4.3] for a version of Dirichlet space on the Bergman tree).

Proposition 3.1. Let $S_{\lambda, q}$ be a Dirichlet shift on $\mathscr{T}=(V, \mathcal{E})$ and let $E:=\operatorname{ker} S_{\lambda, q}^{*}$. Then there exist a z-invariant reproducing kernel Hilbert space $\mathscr{H}_{q}$ of E-valued holomorphic functions defined on the disc $\mathbb{D}$ and a unitary mapping $U: l^{2}(V) \longrightarrow$ $\mathscr{H}_{q}$ such that $\mathscr{M}_{z, q} U=U S_{\lambda, q}$, where $\mathscr{M}_{z, q}$ denotes the operator of multiplication by $z$ on $\mathscr{H}_{q}$. Further, $U$ maps $E$ onto the subspace $\mathscr{E}$ of E-valued constant functions in $\mathscr{H}_{q}$ such that $U g=g$ for every $g \in$ E. Furthermore, we have the following:

(i) the reproducing kernel $\kappa_{\mathscr{H}_{q}}: \mathbb{D} \times \mathbb{D} \rightarrow B(E)$ associated with $\mathscr{H}_{q}$ satisfies $\kappa_{\mathscr{H}_{q}}(\cdot, w) g \in \mathscr{H}_{q}$ and $\left\langle U f, \kappa_{\mathscr{H}_{q}}(\cdot, w) g\right\rangle_{\mathscr{H}_{q}}=\langle(U f)(w), g\rangle_{E}$ for every $f \in$ $l^{2}(V)$ and $g \in E$,

(ii) $\kappa_{\mathscr{H}_{q}}$ is given by

$$
\begin{aligned}
\kappa_{\mathscr{H}_{q}}(z, w) & =\sum_{n=0}^{\infty} \frac{(1)_{n}}{(q)_{n}} z^{n} \bar{w}^{n} P_{\langle\text {eroot }\rangle} \\
& +\sum_{v \in V_{\prec}} \sum_{n=0}^{\infty} \frac{\left(n_{v}+2\right)_{n}}{\left(n_{v}+q+1\right)_{n}} z^{n} \bar{w}^{n} P_{l^{2}(\operatorname{Chi}(v)) \ominus\left\langle\boldsymbol{\lambda}^{v}\right\rangle}(z, w \in \mathbb{D}),
\end{aligned}
$$

where $P_{\mathscr{M}}$ denotes the orthogonal projection of $\mathscr{H}$ onto the subspace $\mathscr{M}$ of $\mathscr{H}$,

(iii) The E-valued polynomials in $z$ are dense in $\mathscr{H}_{q}$. In fact,

$$
\mathscr{H}_{q}=\bigvee\left\{z^{n} f: f \in \mathscr{E}, n \in \mathbb{N}\right\} .
$$

(iv) If $\mathscr{B}$ is an orthonormal basis of $\mathscr{E}$ then $\left\{z^{n} f: f \in \mathscr{B}, n \in \mathbb{N}\right\}$ forms an orthogonal basis of $\mathscr{H}_{q}$.

Remark 3.2. Note that the reproducing kernel Hilbert space $\mathscr{H}_{1}$ is nothing but the vector-valued Hardy space associated with the kernel $\frac{I_{E}}{1-z \bar{w}}(z, w \in \mathbb{D})$, where $I_{E}$ denotes the identity operator on $E$. In view of the decomposition (1.1) of $E$, this is immediate from the result above. 
Proof. The proof is an application of [17, Theorem 2.2]. The first half and parts (i), (iii) follow from [17, Theorem 2.2]. Thus we only need to verify parts (ii) and (iv). Note first that by [17, Theorem 2.2](ii) and Lemma 2.6, $\kappa_{\mathscr{H}_{q}}$ is given by

$$
\kappa_{\mathscr{H}_{q}}(z, w)=I_{E}+\sum_{j, k \geqslant 1} C_{j, k} z^{j} \bar{w}^{k}(z, w \in \mathbb{D})
$$

where $I_{E}$ denotes the identity operator on $E$, and $C_{j, k}$ are bounded linear operators on $E$ given by

$$
C_{j, k}=\left.P_{E} S_{\lambda^{\prime}, q}^{* j} S_{\lambda^{\prime}, q}^{k}\right|_{E}(j, k=1,2, \cdots)
$$

with $P_{E}$ being the orthogonal projection of $l^{2}(V)$ onto $E$. To see that $C_{j, k}=0$ for $j \neq k$, we need the following identity [15, (5.6)]:

$$
\sum_{u \in \mathrm{Chi}^{\langle}\langle k\rangle} \prod_{l=0}^{k-1} \frac{1}{s_{l, u}}=1 \text { for } v \in V \text { and } k \geqslant 1,
$$

where, for a positive integer $l$ and $v \in V, s_{l, v}:=\operatorname{card}\left(\operatorname{sib}\left(\operatorname{par}^{\langle l\rangle}(v)\right)\right)$. This identity for a fixed $v \in V$ can be verified by induction on integers $k \geqslant 1$. Now for $v \in V$ and $j, k \geqslant 1$,

$$
\begin{gathered}
S_{\lambda^{\prime}, q}^{k} e_{v}=\sqrt{\frac{\left(n_{v}+1\right)_{k}}{\left(n_{v}+q\right)_{k}}} \sum_{u \in \mathrm{Chi}^{\prime}\langle k\rangle} \prod_{l=0}^{k-1} \frac{1}{\sqrt{s_{l, u}}} e_{u}, \\
S_{\lambda^{\prime}, q}^{* j} e_{v}= \begin{cases}\sqrt{\frac{\left(n_{v}+q\right)_{-j}}{\left(n_{v}+1\right)_{-j}}} \prod_{l=0}^{j-1} \frac{1}{\sqrt{s_{l, v}}} e_{\text {par }\langle j\rangle(v)} & \text { if } n_{v} \geqslant j, \\
0 & \text { otherwise. }\end{cases}
\end{gathered}
$$

Let $v \in V$ and $j \geqslant k$. Note that if $\operatorname{par}^{\langle j-k\rangle}(v)$ is empty, then $S_{\lambda^{\prime}, q}^{* j} S_{\lambda^{\prime}, q}^{k} e_{v}=0$. Otherwise

$$
\begin{aligned}
S_{\lambda^{\prime}, q}^{* j} S_{\lambda^{\prime}, q}^{k} e_{v} & =\sqrt{\frac{\left(n_{v}+1\right)_{k}}{\left(n_{v}+q\right)_{k}}} \sum_{u \in \mathrm{Chi}^{i k\rangle}(v)} \prod_{l=0}^{k-1} \frac{1}{\sqrt{s_{l, u}}} S_{\lambda^{\prime}, q}^{* j} e_{u} \\
& =\sqrt{\frac{\left(n_{v}+1\right)_{k}}{\left(n_{v}+q\right)_{k}}} \sum_{u \in \mathrm{Chi}^{i k\rangle}(v)}\left(\prod_{l=0}^{k-1} \frac{1}{\sqrt{s_{l, u}}}\right. \\
& \left.\times \sqrt{\frac{\left(n_{u}+q\right)_{-j}}{\left(n_{u}+1\right)_{-j}}} \prod_{m=0}^{j-1} \frac{1}{\sqrt{s_{m, u}}}\right) e_{\mathrm{par}\langle j\rangle}(u) \\
& =\sqrt{\frac{\left(n_{v}+1\right)_{k}}{\left(n_{v}+q\right)_{k}}} \sqrt{\frac{\left(n_{v}+k+q\right)_{-j}}{\left(n_{v}+k+1\right)_{-j}}} \prod_{l=0}^{j-k-1} \frac{1}{\sqrt{s_{l, v}}} e_{\mathrm{par}\langle j-k\rangle(v)},
\end{aligned}
$$

where the last equality follows from (3.2), $n_{u}=n_{v}+k$ and $s_{l, v}=s_{l+k, u}$. This also shows the following:

(a) For $k \in \mathbb{N}$,

$$
S_{\lambda^{\prime}, q}^{* k} S_{\lambda^{\prime}, q}^{k} e_{v}=\frac{\left(n_{v}+1\right)_{k}}{\left(n_{v}+q\right)_{k}} e_{v} .
$$

Here we used the convention that product over the empty set equals 1 .

(b) For non-negative integers $j>k$,

$$
S_{\lambda^{\prime}, q}^{* j} S_{\lambda^{\prime}, q}^{k} e_{v}=\beta(j, k, v) e_{\mathrm{par}}^{\langle j-k\rangle(v)}
$$

for some positive scalar $\beta(j, k, v)$ such that

$$
\beta(j, k, \omega)=\beta(j, k, v) \text { for all } \omega \in \operatorname{sib}(v) .
$$


Let $f \in E$. Note that by (1.1), $f$ takes the form $f=f_{\text {root }}+\sum_{w \in V_{\prec}} f_{w}$, where $f_{\text {root }}=\gamma e_{\text {root }}$ for some $\gamma \in \mathbb{C}$ and $f_{w}=\sum_{v \in \operatorname{Chi}(w)} f(v) e_{v}$ such that

$$
\sum_{v \in \operatorname{Chi}(w)} f(v) \lambda_{v}=0 \text { for } w \in V_{\prec}
$$

In view of (2.1), $\lambda_{v}$ is constant on $\mathrm{Chi}(w)$, and hence we obtain that

$$
\sum_{v \in \operatorname{Chi}(w)} f(v)=0 \text { for all } w \in V_{\prec} .
$$

It follows that for $w \in V_{\prec}$ and $j>k$,

$$
S_{\lambda^{\prime}, q}^{* j} S_{\lambda^{\prime}, q}^{k} f_{w}=\sum_{v \in \operatorname{Chi}(w)} f(v) S_{\lambda^{\prime}, q}^{* j} S_{\lambda^{\prime}, q}^{k} e_{v}=0,
$$

where we used (3.4), (3.5) and (3.6). It may now be concluded from (3.1), (1.1), (3.3) that the reproducing kernel $\kappa_{\mathscr{H}_{q}}$ takes the required form. Note that the conclusion in (3.7) also holds for $S_{\lambda, q}$ (by the same reasoning), and hence the sequence $\left\{z^{n} \mathscr{E}: n \in \mathbb{N}\right\}$ of subspaces of $\mathscr{H}_{q}$ is mutually orthogonal. This combined with part (iii) yields (iv).

Remark 3.3. Note that $\kappa_{\mathscr{H}_{2}}$ takes the form

$$
\begin{aligned}
\kappa_{\mathscr{H}_{2}}(z, w) & =-\frac{1}{z \bar{w}} \log (1-z \bar{w}) P_{\left\langle e_{\text {root }}\right\rangle} \\
& +\sum_{v \in V \prec} \sum_{n=0}^{\infty} \frac{n_{v}+2}{n_{v}+2+n} z^{n} \bar{w}^{n} P_{l^{2}(\operatorname{Chi}(v)) \ominus\left\langle\boldsymbol{\lambda}^{v}\right\rangle} \\
& =-\frac{1}{z \bar{w}} \log (1-z \bar{w}) P_{\left\langle e_{\text {root }}\right\rangle} \\
& -\sum_{v \in V_{\prec}} \frac{n_{v}+2}{z^{n_{v}+1} \bar{w}^{n_{v}+1}}\left(\frac{1}{z \bar{w}} \log (1-z \bar{w})+\sum_{k=0}^{n_{v}} \frac{z^{k} \bar{w}^{k}}{k+1}\right) P_{l^{2}(\operatorname{Chi}(v)) \ominus\left\langle\boldsymbol{\lambda}^{v}\right\rangle}
\end{aligned}
$$

for $z, w \in \mathbb{D} \backslash\{0\}$. A particular case in which $\mathscr{T}$ has only branching point at root, $\kappa \mathscr{H}_{2}$ simplifies to

$$
\begin{aligned}
\kappa_{\mathscr{H}_{2}}(z, w) & =-\frac{1}{z \bar{w}} \log (1-z \bar{w}) P_{\langle\text {eroot }\rangle} \\
& -\frac{2}{z \bar{w}}\left(\frac{1}{z \bar{w}} \log (1-z \bar{w})+1\right) P_{l^{2}(\text { Chi (root) }) \ominus\left\langle\lambda^{\text {root }}\right\rangle}
\end{aligned}
$$

for $z, w \in \mathbb{D} \backslash\{0\}$.

Note that in case $\mathscr{T}$ is a rooted directed tree without any branching vertex (that is, $\mathscr{T}$ is isomorphic to $\mathbb{N}), \mathscr{H}_{q}$ is nothing but the classical Dirichlet space $D_{q}(q \geqslant 1)$, that is, the reproducing kernel Hilbert space associated with reproducing kernel

$$
\sum_{n=0}^{\infty} \frac{(1)_{n}}{(q)_{n}} z^{n} \bar{w}^{n}(z, w \in \mathbb{D}) .
$$

(refer to [20] for the basic theory of classical Dirichlet spaces; the reader is also referred to [7] for an interesting exposition on some recent developments related to Dirichlet spaces). This motivates the following definition.

Definition 3.4. Let $\mathscr{T}=(V, \mathcal{E})$ be a leafless, locally finite rooted directed tree. We refer to the space $\mathscr{H}_{q}$, as constructed in Proposition 3.1, as the Dirichlet space associated with $\mathscr{T}$. 
Corollary 3.5. Let $\mathscr{H}_{q}$ be a Dirichlet space associated with $\mathscr{T}=(V, \mathcal{E})$ and let $\mathscr{M}_{z, q}$ be the operator of multiplication by $z$ on $\mathscr{H}_{q}$. Then, for any $f(z)=\sum_{n=0}^{\infty} f_{n} z^{n}$ in $\mathscr{H}_{q}$, we have

$$
\|f\|_{\mathscr{H}_{q}}^{2}=\sum_{n=0}^{\infty}\left(\left|a_{n}\right|^{2} \frac{(q)_{n}}{(1)_{n}}+\sum_{v \in V_{\prec}}\left\|b_{n, v}\right\|_{l^{2}(V)}^{2} \frac{\left(n_{v}+q+1\right)_{n}}{\left(n_{v}+2\right)_{n}}\right),
$$

where $f_{n}=a_{n} e_{\text {root }}+\sum_{v \in V_{\alpha}} b_{n, v} \in \operatorname{ker} \mathscr{M}_{z, q}^{*}$ is an orthogonal decomposition with $a_{n} \in \mathbb{C}$ and $b_{n, v} \in l^{2}(\operatorname{Chi}(v)) \ominus\left\langle\boldsymbol{\lambda}^{v}\right\rangle$ for every $n \in \mathbb{N}$. Thus $\mathscr{H}_{q}$ is contained in $\mathscr{H}_{1}$.

Proof. To see the first part, in view of Proposition 3.1(iv), it suffices to check that for $f_{n}=a_{n} e_{\text {root }}+\sum_{v \in V_{\prec}} b_{n, v} \in \operatorname{ker} \mathscr{M}_{z, q}^{*}$,

$$
\left\|z^{n} f_{n}\right\|^{2}=\left|a_{n}\right|^{2} \frac{(q)_{n}}{(1)_{n}}+\sum_{v \in V_{\prec}}\left\|b_{n, v}\right\|_{l^{2}(V)}^{2} \frac{\left(n_{v}+q+1\right)_{n}}{\left(n_{v}+2\right)_{n}} .
$$

However, by Proposition 3.1

$$
\left\|z^{n} f_{n}\right\|^{2}=\left\|S_{\lambda, q}^{n} f_{n}\right\|^{2}=\left|a_{n}\right|^{2}\left\|S_{\lambda, q}^{n} e_{\text {root }}\right\|^{2}+\sum_{v \in V_{\prec}}\left\|S_{\lambda, q}^{n} b_{n, v}\right\|^{2},
$$

and hence (3.8) is immediate from Lemma 2.6. The remaining part follows from the inequality $\sum_{n=0}^{\infty}\left(\left|a_{n}\right|^{2}+\sum_{v \in V \prec}\left\|b_{n, v}\right\|_{l^{2}(V)}^{2}\right) \leqslant\|f\|_{\mathscr{H}_{q}}^{2}$ for every $f \in \mathscr{H}_{q}$.

Let $\kappa: \mathbb{D} \times \mathbb{D} \rightarrow B(E)$ be a positive definite kernel such that $\kappa(z, w)$ is invertible for every $z, w \in \mathbb{D}$ and let $\mathscr{H}$ be the reproducing kernel Hilbert space associated with $\kappa$. Following [1, we say that $\mathscr{H}$ has complete Pick property if there exists a positive definite function $F: \mathbb{D} \times \mathbb{D} \rightarrow B(E)$ such that

$$
I_{E}-\kappa(z, w)^{-1}=F(z, w)(z, w \in \mathbb{D}) .
$$

Corollary 3.6. Let $\mathscr{H}_{q}$ be a Dirichlet space associated with $\mathscr{T}=(V, \mathcal{E})$. Then $\mathscr{H}_{q}$ has complete Pick property.

Proof. By Proposition 3.1(ii), $\kappa \mathscr{H}_{q}(z, w)$ is orthogonal direct sum of finitely many positive definite kernels of the form

$$
\kappa_{k, l}(z, w)=\sum_{n=0}^{\infty} \frac{(k)_{n}}{(l)_{n}} z^{n} \bar{w}^{n} P_{k, l},
$$

where $P_{k, l}$ is a non-zero orthogonal projection and $k, l$ are positive integers such that $l \geqslant k$. Thus it suffices to check that $1-\frac{1}{\kappa_{k, l}(z, w)}$ is a positive definite kernel. In view of [1, Theorem 7.33 and Lemma 7.38], it is enough to verify that

$$
\left(\frac{(k)_{n}}{(l)_{n}}\right)^{2} \leqslant \frac{(k)_{n-1}}{(l)_{n-1}} \frac{(k)_{n+1}}{(l)_{n+1}} \text { for every } n \in \mathbb{N} .
$$

However, this is equivalent to $(l+n)(k+n-1) \leqslant(l+n-1)(k+n)(n \in \mathbb{N})$, which is true whenever $l \geqslant k$.

3.1. The vector-valued Dirichlet space $\mathscr{H}_{2}$. It turns out that $\mathscr{H}_{2}$ can be identified with a vector-valued Dirichlet space. Let us first reproduce from [25] the definition of vector-valued Dirichlet spaces.

Let $E$ be a Hilbert space and $\mu$ be a positive $B(E)$-valued measure on the unit circle $\mathbb{T}$. The Poisson integral $P[\mu]: \mathbb{D} \rightarrow B(E)$ of $\mu$ is defined by

$$
P[\mu](z):=\int_{\mathbb{T}} \frac{1-|z|^{2}}{\left|e^{i \theta}-z\right|^{2}} d \mu\left(e^{i \theta}\right)(z \in \mathbb{D}) .
$$


For an analytic function $f: \mathbb{D} \rightarrow E$ of the form $f(z)=\sum_{n=0}^{\infty} f_{n} z^{n}$ with $\left\{f_{n}\right\}_{n \in \mathbb{N}} \subseteq$ $E$, set

$$
\|f\|_{\mu}^{2}:=\sum_{n=0}^{\infty}\left\|f_{n}\right\|_{E}^{2}+\int_{\mathbb{D}}\left\langle P[\mu](z) f^{\prime}(z), f^{\prime}(z)\right\rangle_{E} d A(z),
$$

where $d A$ denotes normalized area measure on $\mathbb{D}$. The E-valued Dirichlet space is defined as

$$
D(\mu):=\left\{f: \mathbb{D} \rightarrow E \mid f \text { is an analytic function such that }\|f\|_{\mu}<\infty\right\} .
$$

Proposition 3.7. Let $\mathscr{H}_{2}$ be a Dirichlet space associated with $\mathscr{T}=(V, \mathcal{E})$ and let $\mathscr{M}_{z, 2}$ be the operator of multiplication by $z$ on $\mathscr{H}_{2}$. Define a positive $B(\mathscr{E})$-valued measure $\mu_{\mathscr{T}}$ given by

$$
d \mu_{\mathscr{T}}\left(e^{i \theta}\right)\left(a e_{\text {root }}+\sum_{v \in V \prec} b_{v}\right):=\left(a e_{\text {root }}+\sum_{v \in V_{\prec}} \frac{b_{v}}{n_{v}+2}\right) d \sigma\left(e^{i \theta}\right),
$$

where $\mathscr{E}:=\operatorname{ker} \mathscr{M}_{z, 2}^{*}, a \in \mathbb{C}, b_{v} \in l^{2}(\operatorname{Chi}(v)) \ominus\left\langle\boldsymbol{\lambda}^{v}\right\rangle$, and $d \sigma$ denotes the normalized arclength measure on unit circle $\mathbb{T}$. Then $\mathscr{H}_{2}$ equals the $\mathscr{E}$-valued Dirichlet space $D\left(\mu_{\mathscr{T}}\right)$ with equality of norms.

Proof. We claim that if $f \in \mathscr{H}_{2}$ then $\|f\|_{\mathscr{H}_{2}}=\|f\|_{\mu_{\mathscr{T}}}$. Note that by Corollary 3.5.

$$
\begin{aligned}
\|f\|_{\mathscr{H}_{2}}^{2} & =\sum_{n=0}^{\infty}\left((n+1)\left|a_{n}\right|^{2}+\sum_{v \in V_{\prec}}\left(1+\frac{n}{n_{v}+2}\right)\left\|b_{n, v}\right\|_{E}^{2}\right) \\
& =\|f\|_{\mathscr{H}_{1}}^{2}+\sum_{n=0}^{\infty} n\left(\left|a_{n}\right|^{2}+\sum_{v \in V_{\prec}} \frac{\left\|b_{n, v}\right\|_{E}^{2}}{n_{v}+2}\right) .
\end{aligned}
$$

In view of Proposition 3.1, it suffices to verify $\left\|f_{n}\right\|_{\mathscr{H}_{2}}=\left\|f_{n}\right\|_{\mu_{\mathscr{T}}}$, where

$$
f_{n}(z)=\left(a e_{\text {root }}+\sum_{v \in V_{\prec}} b_{v}\right) z^{n}(n \geqslant 1) .
$$

However, in the light of (3.9) and (3.11), it is enough to verify that

$$
n\left(|a|^{2}+\sum_{v \in V_{\prec}} \frac{\left\|b_{v}\right\|_{l^{2}(V)}^{2}}{n_{v}+2}\right)=\int_{\mathbb{D}}\left\langle P\left[\mu_{\mathscr{T}}\right](z) f_{n}^{\prime}(z), f_{n}^{\prime}(z)\right\rangle_{E} d A(z),
$$

where $f_{n}^{\prime}(z)=\left(a e_{\text {root }}+\sum_{v \in V_{\prec}} b_{v}\right) n z^{n-1}$ for integers $n \geqslant 1$. Note first that for $z \in \mathbb{D}$,

$$
\begin{aligned}
P\left[\mu_{\mathscr{T}}\right](z)\left(a e_{\text {root }}+\sum_{v \in V_{\prec}} b_{v}\right) & =P[\sigma](z)\left(a e_{\text {root }}+\sum_{v \in V_{\prec}} \frac{b_{v}}{n_{v}+2}\right) \\
& =a e_{\text {root }}+\sum_{v \in V_{\prec}} \frac{b_{v}}{n_{v}+2} .
\end{aligned}
$$


Since $\|g\|_{\mathscr{H}_{2}}=\|g\|_{l^{2}(V)}$ for every $g \in \mathscr{E}$ (Proposition 3.1), it now follows that

$$
\begin{aligned}
& \int_{\mathbb{D}}\left\langle P\left[\mu_{\mathscr{T}}\right](z) f_{n}^{\prime}(z), f_{n}^{\prime}(z)\right\rangle_{E} d A(z) \\
= & \int_{\mathbb{D}} n^{2}\left|z^{n-1}\right|^{2}\left\langle a e_{\text {root }}+\sum_{v \in V_{\prec}} \frac{b_{v}}{n_{v}+2}, a e_{\text {root }}+\sum_{v \in V_{\prec}} b_{v}\right\rangle_{E} d A(z) \\
= & \left(|a|^{2}+\sum_{v \in V_{\prec}} \frac{\left\|b_{v}\right\|_{\mathscr{H}_{2}}^{2}}{n_{v}+2}\right) \int_{\mathbb{D}} n^{2}\left|z^{n-1}\right|^{2} d A(z) \\
= & n\left(|a|^{2}+\sum_{v \in V_{\prec}} \frac{\left\|b_{v}\right\|_{l^{2}(V)}^{2}}{n_{v}+2}\right) .
\end{aligned}
$$

Thus the claim stands verified. The claim implies in particular that $\mathscr{H}_{2} \subseteq D\left(\mu_{\mathscr{T}}\right)$. Since $E$-valued analytic polynomials are dense in $D\left(\mu_{\mathscr{T}}\right)([25$, Corollary 3.1$])$, we must have $\mathscr{H}_{2}=D\left(\mu_{\mathscr{T}}\right)$.

As in the classical case [20, Corollary 1.4.3], any Dirichlet space $\mathscr{H}_{2}$ associated with $\mathscr{T}$ admits the conformal invariance property.

Corollary 3.8. Let $\mathscr{H}_{2}$ be a Dirichlet space associated with $\mathscr{T}=(V, \mathcal{E})$ and let $\phi$ be an automorphism of the unit disc. Then, for every $f \in \mathscr{H}_{2}, f \circ \phi \in \mathscr{H}_{2}$ and

$\int_{\mathbb{D}}\left\langle P\left[\mu_{\mathscr{T}}\right](z)(f \circ \phi)^{\prime}(z),(f \circ \phi)^{\prime}(z)\right\rangle_{E} d A(z)=\int_{\mathbb{D}}\left\langle P\left[\mu_{\mathscr{T}}\right](z) f^{\prime}(z), f^{\prime}(z)\right\rangle_{E} d A(z)$,

where $\mu_{\mathscr{T}}$ is as given in (3.10).

Proof. Let $f \in \mathscr{H}_{2}$. Note that by change of variables,

$$
\begin{aligned}
& \int_{\mathbb{D}}\left\langle P\left[\mu_{\mathscr{T}}\right](z)(f \circ \phi)^{\prime}(z),(f \circ \phi)^{\prime}(z)\right\rangle_{E} d A(z) \\
= & \int_{\mathbb{D}}\left\langle P\left[\mu_{\mathscr{T}}\right](z) f^{\prime}(\phi(z)), f^{\prime}(\phi(z))\right\rangle_{E}\left|\phi^{\prime}(z)\right|^{2} d A(z) \\
\stackrel{\text { 3.12 }}{=} & \int_{\mathbb{D}}\left\langle P\left[\mu_{\mathscr{T}}\right](w) f^{\prime}(w), f^{\prime}(w)\right\rangle_{E} d A(w),
\end{aligned}
$$

which completes the proof of the second part. The first part now follows from Littlewood's Theorem [27, Chapter 1].

We present below a proof of the special case $q=2$ of Theorem 2.4, which exploits the theory of vector-valued Dirichlet spaces [25].

Proof of Theorem 2.4 (Case $q=2$ ). For $j=1,2$, let $\mathscr{H}_{2}^{(j)}$ be the Dirichlet space associated with $\mathscr{T}_{j}=\left(V_{j}, \mathcal{E}_{j}\right)$ and let $\mathscr{M}_{z, 2}^{(j)}$ be the operator of multiplication by $z$ on $\mathscr{H}_{2}^{(j)}$. In view of Proposition 3.1 it suffices to check that $\mathscr{M}_{z, 2}^{(1)}$ is unitarily equivalent to $\mathscr{M}_{z, 2}^{(2)}$ if and only if (2.3) holds for every $n \in \mathbb{N}$. By the preceding proposition and [25, Theorem 4.2], the multiplication operator $\mathscr{M}_{z, 2}^{(1)}$ on $D\left(\mu_{\mathscr{T}_{1}}\right)$ is unitarily equivalent to the multiplication operator $\mathscr{M}_{z, 2}^{(2)}$ on $D\left(\mu_{\mathscr{T}_{2}}\right)$ if and only if there exists a unitary map $U: \mathscr{E}_{1} \rightarrow \mathscr{E}_{2}$ such that $\mu_{\mathscr{T}_{1}}(A)=U^{*} \mu_{\mathscr{T}_{2}}(A) U$ for every Borel subset $A$ of the unit circle $\mathbb{T}$ (see (3.10). However, by (3.10), this happens if and only if the diagonal matrices $\oplus_{v \in V_{\prec}^{(1)}} \alpha_{v} I_{\beta_{v, 1}}$ and $\oplus_{v \in V_{\prec}^{(2)}} \alpha_{v} I_{\beta_{v, 2}}$ are unitarily equivalent, where $I_{m}$ denotes the $m \times m$ identity matrix and

$$
\alpha_{v}:=\frac{1}{n_{v}+2}, \quad \beta_{v, j}:=\operatorname{card}(\operatorname{Chi}(v))-1(j=1,2) .
$$


The later one holds if and only if $\oplus_{v \in V_{\prec}^{(1)}} \alpha_{v} I_{\beta_{v, 1}}$ and $\oplus_{v \in V_{\prec}^{(2)}} \alpha_{v} I_{\beta_{v, 2}}$ have the same eigenvalues counted with multiplicity. However, $\sum_{v \in V_{\prec}^{(j)} \cap \mathcal{G}_{n}^{(1)}}(\operatorname{card}(\operatorname{Chi}(v))-1)$ is the multiplicity of the eigenvalue $\frac{1}{n+2}$ for $j=1,2$. The desired equivalence is now immediate.

\section{Bergman Spaces Associated with Directed Trees}

It is evident from the proof of Proposition 3.1 that one can also associate a functional Hilbert space, say, $\mathscr{H}_{-q}$ with the Cauchy dual $S_{\lambda^{\prime}, q}$ of the Dirichlet shift $S_{\lambda, q}$. In particular, $\mathscr{H}_{-q}$ is a reproducing kernel Hilbert space associated with the kernel $\kappa_{\mathscr{H}_{-q}}$ given by

$$
\begin{aligned}
\kappa_{\mathscr{H}_{-q}}(z, w) & =\sum_{n=0}^{\infty} \frac{(q)_{n}}{(1)_{n}} z^{n} \bar{w}^{n} P_{\left\langle e_{\text {root }}\right\rangle} \\
& +\sum_{v \in V \prec} \sum_{n=0}^{\infty} \frac{\left(n_{v}+q+1\right)_{n}}{\left(n_{v}+2\right)_{n}} z^{n} \bar{w}^{n} P_{l^{2}(\operatorname{Chi}(v)) \ominus\left\langle\boldsymbol{\lambda}^{v}\right\rangle}(z, w \in \mathbb{D}) .
\end{aligned}
$$

This has been recorded in [15, Proposition 5.1.8]. It is equally clear that $S_{\lambda^{\prime}, q}$ is unitarily equivalent to the operator of multiplication by $z$ on $\mathscr{H}_{-q}$.

Definition 4.1. Let $q$ be an integer bigger than 1. Let $\mathscr{T}=(V, \mathcal{E})$ be a leafless, locally finite rooted directed tree. We refer to $\mathscr{H}_{-q}$ as the Bergman space associated with $\mathscr{T}$.

Remark 4.2. Note that in case $\mathscr{T}$ is a rooted directed tree without any branching vertex, $\mathscr{H}_{-q}$ is nothing but the classical Bergman space $B_{q}(q \geqslant 2)$, that is, the reproducing kernel Hilbert space associated with

$$
\sum_{n=0}^{\infty} \frac{(q)_{n}}{(1)_{n}} z^{n} \bar{w}^{n}(z, w \in \mathbb{D})
$$

(refer to 21] for the basic theory of classical Bergman spaces).

Lemma 4.3. Let $\mathscr{H}_{-q}$ be a Bergman space associated with $\mathscr{T}=(V, \mathcal{E})$ and let $\mathscr{M}_{z,-q}$ be the operator of multiplication by $z$ on $\mathscr{H}_{-q}$. Then for $f(z)=\sum_{n=0}^{\infty} f_{n} z^{n}$ in $\mathscr{H}_{-q}$ with $f_{n}=a_{n} e_{\text {root }}+\sum_{v \in V} b_{n, v} \in \operatorname{ker} \mathscr{M}_{z,-q}^{*}, a_{n} \in \mathbb{C}$ and $b_{n, v} \in l^{2}(\operatorname{Chi}(v)) \ominus\left\langle\boldsymbol{\lambda}^{v}\right\rangle$ for every $n \in \mathbb{N}$, we have the following:

(i) $\|f\|_{\mathscr{H}_{-q}}^{2}=\sum_{n=0}^{\infty}\left(\left|a_{n}\right|^{2} \frac{(1)_{n}}{(q)_{n}}+\sum_{v \in V}\left\|b_{n, v}\right\|_{l^{2}(V)}^{2} \frac{\left(n_{v}+2\right)_{n}}{\left(n_{v}+q+1\right)_{n}}\right)$.

(ii) if $q \geqslant 2$, then

$$
\|f\|_{\mathscr{H}_{-q}}^{2}=\int_{\mathbb{D}}\left\langle d \nu_{\mathscr{T}}(z) f(z), f(z)\right\rangle=\sum_{n=0}^{\infty} \int_{\mathbb{D}}\left|z^{n}\right|^{2}\left\langle d \nu_{\mathscr{T}}(z) f_{n}, f_{n}\right\rangle,
$$

where

$$
d \nu_{\mathscr{T}}(z)\left(a e_{\text {root }}+\sum_{v \in V_{\prec}} b_{v}\right):=\left(a w_{0}(z) e_{\text {root }}+\sum_{v \in V_{\prec}} w_{n_{v}+1}(z) b_{v}\right) d A(z)
$$

with $d A$ denoting the normalized area measure on unit disc $\mathbb{D}$ and

$$
w_{l}(z)=(l+1) \cdots(l+q-1) \sum_{i=1}^{q-1} \frac{|z|^{2(i+l-1)}}{\prod_{1 \leqslant j \neq i \leqslant q-1}(j-i)}(z \in \mathbb{D}, l \in \mathbb{N}) .
$$

In particular, $\mathscr{H}_{-q}$ equals $L_{a}^{2}\left(\nu_{\mathscr{T}}\right)$, that is, the closure of E-valued analytic polynomials in $L^{2}\left(\nu_{\mathscr{T}}\right)$, with equality of norms. 
Proof. The first part can be obtained along the lines of the proof of Corollary [3.5], The first equality in the second part may be deduced from (i) and [5. Corollary 3.8] while the second one is an immediate consequence of the fact that

$$
\int_{\mathbb{D}} z^{n} \bar{z}^{m} w(|z|) d A(z)=\delta_{m n}(m, n \in \mathbb{N})
$$

for any continuous function $w:[0,1] \rightarrow(0, \infty)$.

The Bergman spaces $\mathscr{H}_{-q}$ can be realized as Hilbert modules over the polynomial ring $\mathbb{C}[z]$ with module action given by

$$
(p, f) \in \mathbb{C}[z] \times \mathscr{H}_{-q} \longmapsto p(z) f \in \mathscr{H}_{-q} .
$$

A family of Hilbert modules $\mathcal{H}_{N}^{(\eta, Y)}$ (vector-valued analogs of the reproducing kernel Hilbert spaces associated with the kernel $\frac{1}{(1-z \bar{w})^{q}}, q>0$ ) have been introduced in 24 in the context of classification of homogeneous operators within the class of Cowen-Douglas operators (see the discussion prior to [24, Theorem 3.1] for the exact description of the spaces $\left.\mathcal{H}_{N}^{(\eta, Y)}\right)$. Recall that a bounded linear operator $T$ on $\mathcal{H}$ is homogeneous if for every automorphism $\phi$ of the open unit disc $\mathbb{D}, \phi(T)$ is unitarily equivalent to $T$. Note that the assumption that $\phi(T)$ is well-defined as a bounded linear operator on $\mathcal{H}$ is a part of the definition of the homogeneous operator. Also, the multiplication operator $\mathscr{M}_{z,-q}$ on the classical Bergman space $B_{q}$ provides an example of homogeneous operator [9, Theorem 5.2].

We say that the Hilbert module $\mathscr{H}_{-q}$ over $\mathbb{C}[z]$ is homogeneous if $\mathscr{M}_{z,-q}$ is homogeneous. The natural question arises here is that for which directed trees $\mathscr{T}, \mathscr{H}_{-q}$ is a homogeneous Hilbert module? The second question arises is whether $\mathscr{H}_{-q}$ and $\mathcal{H}_{N}^{(\eta, Y)}$ are unitarily equivalent as Hilbert modules over $\mathbb{C}[z]$ ? Both these questions can be answered with the help of [24, Theorem 4.1] and the following general fact.

Proposition 4.4. Let $\mathscr{H}_{-q}$ be a Bergman space associated with the directed tree $\mathscr{T}=(V, \mathcal{E})$ of finite branching index. Then the following statements are equivalent:

(i) $\mathscr{H}_{-q}$ is a homogeneous Hilbert module.

(ii) $\mathscr{T}$ is isomorphic to $\mathbb{N}$.

Proof. Let $\phi$ be an automorphism of the open unit disc $\mathbb{D}$. Since $\mathscr{M}_{z,-q}$ is of spectral radius 1 (Lemma 2.6) and since $\kappa(\cdot, w) g$ provides eigenvector for $\mathscr{M}_{z,-q}^{*}$ with corresponding eigenvalue $\bar{w} \in \mathbb{D}$, the spectrum of $\mathscr{M}_{z,-q}$ equals the closed unit disc $\overline{\mathbb{D}}$. In particular, $\mathscr{M}_{\phi, q}:=\phi\left(\mathscr{M}_{z,-q}\right)$ makes sense.

In case $\mathscr{T}$ is isomorphic to $\mathbb{N}$, the implication (ii) $\Longrightarrow$ (i) is immediate from 9 , Theorem 5.2].

To see that (i) implies (ii), suppose there exists a unitary $U: \mathscr{H}_{-q} \rightarrow \mathscr{H}_{-q}$ such that $U \mathscr{M}_{z,-q}=\mathscr{M}_{\phi, q} U$. Let $\left\{g_{j}: j=1, \cdots, d\right\}$ be an orthonormal basis for $E:=\operatorname{ker} \mathscr{M}_{z,-q}^{*}$, where $g_{1}:=e_{\text {root }}$ and $d$ is finite. One may conclude from (3.3) and (3.7) that for $1 \leqslant i \neq j \leqslant d$, the sequences $\left\{z^{n} g_{i}\right\}_{n \in \mathbb{N}}$ and $\left\{z^{n} g_{j}\right\}_{n \in \mathbb{N}}$ are mutually orthogonal. This yields the decomposition $\mathscr{H}_{-q}=\oplus_{j=1}^{d} \mathscr{H}_{j}$, where

$$
\mathscr{H}_{j}=\bigvee\left\{z^{n} g_{j}: n \in \mathbb{N}\right\}(j=1, \cdots, d) .
$$

Since $\mathscr{H}_{1}, \cdots, \mathscr{H}_{d}$ are $z$-invariant subspaces of $\mathscr{H}_{-q}, \mathscr{M}_{z,-q}=\oplus_{j=1}^{d} \mathscr{M}_{z,-q}^{(j)}$, where $\mathscr{M}_{z,-q}^{(j)}=\left.\mathscr{M}_{z,-q}\right|_{\mathscr{H}_{j}}$ for $j=1, \cdots, d$. On the other hand, by [17, Corollary 5.6], the Hilbert space adjoint of $\mathscr{M}_{z,-q}$ belongs to the Cowen-Douglas class $B_{d}(\mathbb{D})$ (the reader is referred to [19] for the definition of Cowen-Douglas class $\left.B_{d}(\cdot)\right)$. Also, the Hilbert space adjoint of $\mathscr{M}_{z,-q}^{(j)}$ belongs to $B_{1}(\mathbb{D})$ for every $j=1, \cdots, d$. Since the classification of homogeneous operators in $B_{d}(\mathbb{D})$ is the same as that of the 
corresponding homogeneous holomorphic Hermitian bundles defined on $\mathbb{D}[11$, Section 1], we may conclude from [24, Corollary 2.1] that $\mathscr{M}_{z,-q}^{(j)}$ must be homogeneous for every $j=1, \cdots, d$. However, by [9, List 3.1], this is possible only if for every $j=1, \cdots, d$, there exist $a_{j}>0$ such that $\mathscr{M}_{z,-q}^{(j)}$ is unitarily equivalent to the operator $M_{z}^{(j)}$ of multiplication by $z$ on a reproducing kernel Hilbert space $H_{j}$ associated with the kernel $\frac{1}{(1-z \bar{w})^{a_{j}}}(z, w \in \mathbb{D})$. For $j=1, \cdots, d$, let $U_{j}: H_{j} \rightarrow \mathscr{H}_{j}$ be the unitary operator such that

$$
\mathscr{M}_{z,-q}^{(j)} U_{j}=U_{j} M_{z}^{(j)}
$$

Note that $U_{j}$ maps $\operatorname{ker}\left(M_{z}^{(j)}\right)^{*}$ onto $\operatorname{ker}\left(\mathscr{M}_{z,-q}^{(j)}\right)^{*}$. Note that $g:=U_{j}(1 /\|1\|) \in$ $\operatorname{ker}\left(\mathscr{M}_{z,-q}^{(j)}\right)^{*}$. If $d=1$ then clearly $\mathscr{T}$ is isomorphic to $\mathbb{N}$. Suppose the contrary. Then $g \neq e_{\text {root }}$ for some $j=1, \cdots, d$. Thus $g=\sum_{w \in \operatorname{Chi}(v)} \alpha_{w} e_{w} \in l^{2}(\operatorname{Chi}(v)) \ominus\left\langle\boldsymbol{\lambda}^{v}\right\rangle$ for some $v \in V_{\prec}$. Hence, for any integer $k \geqslant 1$, by Lemma 2.6.

$$
\left\|\left(\mathscr{M}_{z,-q}^{(j)}\right)^{k} g\right\|^{2}=\sum_{w \in \operatorname{Chi}(v)}\left|\alpha_{w}\right|^{2} \frac{\left(n_{w}+1\right)_{k}}{\left(n_{w}+q\right)_{k}}=\frac{\left(n_{v}+2\right)_{k}}{\left(n_{v}+q+1\right)_{k}} .
$$

On the other hand,

$$
\left\|\left(\mathscr{M}_{z,-q}^{(j)}\right)^{k} g\right\|^{2}=\left\|\left(M_{z}^{(j)}\right)^{k}(1 /\|1\|)\right\|^{2}=\frac{(1)_{k}}{\left(a_{j}\right)_{k}}
$$

After combining last two equations for $k=1,2$, we obtain $n_{v}+1=0$, which is absurd. Hence $d=1$, and we obtain (ii).

The proof of the preceding proposition actually yields the following general fact.

Corollary 4.5. Let $S_{\lambda}$ be a left-invertible, homogeneous weighted shift on $\mathscr{T}$ with finite dimensional cokernel $E:=\operatorname{ker} S_{\lambda}^{*}$. If $\left\{g_{j}: j=1, \cdots, d\right\}$ is an orthonormal basis of $E$ such that for $1 \leqslant i \neq j \leqslant d$, the sequences $\left\{S_{\lambda}^{n} g_{i}\right\}_{n \in \mathbb{N}}$ and $\left\{S_{\lambda}^{n} g_{j}\right\}_{n \in \mathbb{N}}$ are mutually orthogonal, then there exists a $>0$ such that $S_{\lambda}$ is unitarily equivalent to the operator of multiplication by $z$ on a reproducing kernel Hilbert space associated with the kernel $\frac{1}{(1-z \bar{w})^{a}}(z, w \in \mathbb{D})$.

We do not know whether a rooted directed tree, which is non-isomorphic to $\mathbb{N}$, supports a homogeneous weighted shift.

\section{Classification of Dirichlet Shifts}

For the sake of convenience, we reproduce the statement of Theorem 2.4 from Section 2.

Theorem 5.1. Let $q$ be an integer bigger than 1 . For $j=1,2$, let $S_{\lambda, q}^{(j)}$ be the Dirichlet shift on rooted directed tree $\mathscr{T}_{j}=\left(V_{j}, \mathcal{E}_{j}\right)$ with $\operatorname{root}_{j}$, let $\mathcal{G}_{n}^{(j)}:=\left\{v \in V_{j}\right.$ : $\left.v \in \operatorname{Chi}^{\langle n\rangle}\left(\operatorname{root}_{j}\right)\right\}(n \in \mathbb{N})$, and $E_{j}=\operatorname{ker}\left(S_{\lambda, q}^{(j)}\right)^{*}$. Then $S_{\lambda, q}^{(1)}$ is unitarily equivalent to $S_{\lambda, q}^{(2)}$ if and only if for every $n \in \mathbb{N}$,

$$
\sum_{v \in V_{\prec}^{(1)} \cap \mathcal{G}_{n}^{(1)}}(\operatorname{card}(\operatorname{Chi}(v))-1)=\sum_{v \in V_{\prec}^{(1)} \cap \mathcal{G}_{n}^{(2)}}(\operatorname{card}(\operatorname{Chi}(v))-1) .
$$

Proof. Note that $S_{\lambda, q}^{(1)}$ is unitarily equivalent to $S_{\lambda, q}^{(2)}$ if and only if $S_{\lambda^{\prime}, q}^{(1)}$ is unitarily equivalent to $S_{\lambda^{\prime}, q}^{(2)}$. Hence, in view of Lemma 4.3, it suffices to check that the multiplication operator $\mathscr{M}_{z, q}^{(1)}$ on $L_{a}^{2}\left(\nu_{\mathscr{T}_{1}}\right)$ is unitarily equivalent to the multiplication operator $\mathscr{M}_{z, q}^{(2)}$ on $L_{a}^{2}\left(\nu_{\mathscr{T}_{2}}\right)$ if and only if (5.1) holds for every $n \in \mathbb{N}$. 
Let $U: L_{a}^{2}\left(\nu_{\mathscr{T}_{1}}\right) \rightarrow L_{a}^{2}\left(\nu_{\mathscr{T}_{2}}\right)$ be a unitary map such that

$$
U \mathscr{M}_{z, q}^{(1)}=\mathscr{M}_{z, q}^{(2)} U
$$

Note that $U$ maps $\operatorname{ker}\left(\mathscr{M}_{z, q}^{(1)}\right)^{*}$ unitarily onto $\operatorname{ker}\left(\mathscr{M}_{z, q}^{(2)}\right)^{*}$. For $g, h \in \operatorname{ker}\left(\mathscr{M}_{z, q}^{(1)}\right)^{*}$, define a signed finite measure $\mu_{g, h}$ on the open unit disc $\mathbb{D}$ by

$$
\mu_{g, h}(\sigma)=\int_{\sigma}\left\langle\left(U^{*} d \nu_{\mathscr{T}_{2}}(z) U-d \nu_{\mathscr{T}_{1}}(z)\right) g, h\right\rangle \text { for a Borel subset } \sigma \text { of } \mathbb{D} \text {. }
$$

Note that

$$
\begin{aligned}
\int_{\mathbb{D}} z^{n} \bar{z}^{m}\left\langle U^{*} d \nu_{\mathscr{T}_{2}}(z) U g, h\right\rangle & =\left\langle z^{n} U g, z^{m} U h\right\rangle_{L_{a}^{2}\left(\nu_{\mathscr{T}_{2}}\right)} \stackrel{\sqrt[5.2]{=}}{=}\left\langle U z^{n} g, U z^{m} h\right\rangle_{L_{a}^{2}\left(\nu_{\mathscr{T}_{2}}\right)} \\
& =\left\langle z^{n} g, z^{m} h\right\rangle_{L_{a}^{2}\left(\nu_{\mathscr{T}_{1}}\right)}=\int_{\mathbb{D}} z^{n} \bar{z}^{m}\left\langle d \nu_{\mathscr{T}_{1}}(z) g, h\right\rangle .
\end{aligned}
$$

It follows that $\int_{\mathbb{D}} p(z, \bar{z}) d \mu_{g, h}=0$ for any polynomial $p$ in $z$ and $\bar{z}$. By StoneWeierstrass and Riesz Representation Theorems, $\mu_{g, h}$ is identically 0. Since this holds for arbitrary choices of $g, h$ in $\operatorname{ker}\left(\mathscr{M}_{z, q}^{(1)}\right)^{*}$, we conclude that

$$
U^{*} \nu_{\mathscr{T}_{2}}(\sigma) U=\nu_{\mathscr{T}_{1}}(\sigma) \text { for every Borel subset } \sigma \text { of } \mathbb{D} \text {. }
$$

It is now clear from (4.1) that for every Borel subset $\sigma$ of $\mathbb{D}, a \in \mathbb{C}$, and $b_{v} \in$ $l^{2}(\operatorname{Chi}(v)) \ominus\left\langle\boldsymbol{\lambda}^{v}\right\rangle\left(v \in V_{\prec}^{(1)}\right)$,

$$
\begin{aligned}
& \nu_{\mathscr{T}_{2}}(\sigma) U\left(a e_{\text {root }_{1}}+\sum_{v \in V_{\prec}^{(1)}} b_{v}\right) \\
= & U \int_{\sigma}\left(a w_{0}(z) e_{\text {root }_{1}}+\sum_{v \in V_{\prec}^{(1)}} w_{n_{v}+1}(z) b_{v}\right) d A(z) .
\end{aligned}
$$

Since $U e_{\text {root }_{1}} \in \operatorname{ker}\left(\mathscr{M}_{z, q}^{(2)}\right)^{*}$, there exist $a^{\prime} \in \mathbb{C}$, and $b_{v}^{\prime} \in l^{2}(\operatorname{Chi}(v)) \ominus\left\langle\boldsymbol{\lambda}^{v}\right\rangle$ for $v \in V_{\prec}^{(2)}$ such that

$$
U e_{\text {root }_{1}}=a^{\prime} e_{\text {root }_{2}}+\sum_{v \in V_{\prec}^{(2)}} b_{v}^{\prime} .
$$

Letting $a=1$ and $b_{v}=0$ for all $v \in V_{\prec}^{(1)}$ in (5.4), we obtain

$$
\begin{aligned}
\left(\int_{\sigma} w_{0}(z) d A(z)\right)\left(a^{\prime} e_{\text {root }_{2}}+\sum_{v \in V_{\prec}^{(2)}} b_{v}^{\prime}\right) & =\left(\int_{\sigma} w_{0}(z) d A(z)\right) U e_{\text {root }_{1}} \\
& \stackrel{(5.4)}{=} \nu_{\mathscr{T}_{2}}(\sigma) U e_{\text {root }_{1}} \\
& \stackrel{\text { (4.1) }}{=} \int_{\sigma}\left(a^{\prime} w_{0}(z) d A(z)\right) e_{\text {root }_{2}} \\
& +\sum_{v \in V_{\prec}^{(2)}} \int_{\sigma}\left(w_{n_{v}+1}(z) d A(z)\right) b_{v}^{\prime}
\end{aligned}
$$

for every Borel subset $\sigma$ of $\mathbb{D}$. Since for some Borel set $\sigma, \int_{\sigma} w_{0}(z) d A(z) \neq$ $\int_{\sigma} w_{l}(z) d A(z)$ for any positive integer $l$, by comparing coefficients on both sides, we obtain $b_{v}^{\prime}=0$ for every $v \in V_{\prec}^{(2)}$, and hence $U e_{\text {root }_{1}}=a^{\prime} e_{\text {root }_{2}}$ with $\left|a^{\prime}\right|=1$. This shows that

$$
U\left(\left\langle e_{\text {root }_{1}}\right\rangle\right)=\left\langle e_{\text {root }_{2}}\right\rangle
$$


Since $U$ maps $\operatorname{ker}\left(\mathscr{M}_{z, q}^{(1)}\right)^{*}$ unitarily onto $\operatorname{ker}\left(\mathscr{M}_{z, q}^{(2)}\right)^{*}$, we obtain

$$
\left.U\left(\bigoplus_{v \in V_{\prec}^{(1)}} l^{2}(\operatorname{Chi}(v)) \ominus\left\langle\boldsymbol{\lambda}^{v}\right\rangle\right)\right)=\bigoplus_{v \in V_{\prec}^{(2)}} l^{2}(\operatorname{Chi}(v)) \ominus\left\langle\boldsymbol{\lambda}^{v}\right\rangle .
$$

Next, since $U b_{v_{0}} \in \operatorname{ker}\left(\mathscr{M}_{z, q}^{(2)}\right)^{*}$ for a fixed $v_{0} \in V_{\prec}^{(1)}$, there exist $b_{v}^{\prime} \in l^{2}(\operatorname{Chi}(v)) \ominus$ $\left\langle\boldsymbol{\lambda}^{v}\right\rangle$ for $v \in V_{\prec}^{(2)}$ such that

$$
U b_{v_{0}}=\sum_{v \in V_{\prec}^{(2)}} b_{v}^{\prime} .
$$

As in the preceding paragraph, one may let $a=0$ and $b_{v}=0$ for all $v \in V_{\prec}^{(1)} \backslash\left\{v_{0}\right\}$ in (5.4) to obtain

$$
\left(\int_{\sigma} w_{n_{v_{0}}+1}(z) d A(z)\right)\left(\sum_{v \in V_{\prec}^{(2)}} b_{v}^{\prime}\right)=\sum_{v \in V_{\prec}^{(2)}} \int_{\sigma}\left(w_{n_{v}+1}(z) d A(z)\right) b_{v}^{\prime} .
$$

Since for some Borel set $\sigma, \int_{\sigma} w_{l}(z) d A(z) \neq \int_{\sigma} w_{m}(z) d A(z)$ for positive integers $l \neq m$, by comparing coefficients on both sides, we obtain $b_{v}^{\prime}=0$ for every $v \in$ $V_{\prec}^{(2)} \cap \mathcal{G}_{n}^{(2)}$ with $n \neq n_{v_{0}}$, and hence $U b_{v_{0}}=\sum_{v \in V_{\prec}^{(2)} \cap \mathcal{G}_{n_{v_{0}}}} b_{v}^{\prime}$. Since $v_{0}$ is arbitrary, by (15.5), $U$ must map $W_{1}^{(n)}$ injectively onto $W_{2}^{(n)}$ for every $n \in \mathbb{N}$, where

$$
W_{j}^{(n)}=\bigoplus_{v \in V_{\prec}^{(j)} \cap \mathcal{G}_{n}^{(j)}} l^{2}(\operatorname{Chi}(v)) \ominus\left\langle\boldsymbol{\lambda}^{v}\right\rangle, \quad n \in \mathbb{N}, j=1,2 .
$$

Thus for every $n \in \mathbb{N}$,

$$
\sum_{v \in V_{\prec}^{(1)} \cap \mathcal{G}_{n}^{(1)}}(\operatorname{card}(\operatorname{Chi}(v))-1)=\sum_{v \in V_{\prec}^{(2)} \cap \mathcal{G}_{n}^{(2)}}(\operatorname{card}(\operatorname{Chi}(v))-1) .
$$

Conversely, suppose (5.1) holds for every $n \in \mathbb{N}$. For $n \in \mathbb{N}$ and $j=1,2$, let

$$
\mathcal{M}_{j, 0}=\left\langle e_{\text {root }_{j}}\right\rangle, \quad \mathcal{M}_{j, n}=\bigoplus_{v \in V_{\prec}^{(1)} \cap \mathcal{G}_{n}^{(j)}} l^{2}(\operatorname{Chi}(v)) \ominus\left\langle\boldsymbol{\lambda}^{v}\right\rangle .
$$

By (5.1), we must have $\operatorname{dim} \mathcal{M}_{1, n}=\operatorname{dim} \mathcal{M}_{2, n}$ for every $n \in \mathbb{N}$. Let $U=\oplus_{n \in \mathbb{N}} U_{n}$, where $U_{n}$ is a unitary from $\mathcal{M}_{1, n}$ onto $\mathcal{M}_{2, n}$ for $n \in \mathbb{N}$. Further, we can choose $U_{0}$ such that $U e_{\text {root }_{1}}=e_{\text {root }_{2}}$. Note that $U$ is a unitary from $\operatorname{ker}\left(\mathscr{M}_{z, q}^{(1)}\right)^{*}$ onto $\operatorname{ker}\left(\mathscr{M}_{z, q}^{(2)}\right)^{*}$. We verify that $U$ satisfies (5.3). It suffices to check that

$$
U^{*} \nu_{\mathscr{T}_{2}}(\sigma) U f=\nu_{\mathscr{T}_{1}}(\sigma) f
$$

where $f=\alpha e_{\text {root }_{1}}+\beta b_{v, n}$ for $\alpha, \beta \in \mathbb{C}$ and $\left\{b_{v, n}\right\}$ is an orthonormal basis for $\mathcal{M}_{1, n}$ for integers $n \geq 1$. Indeed, for a Borel subset $\sigma$ of $\mathbb{D}$,

$$
\begin{aligned}
U^{*} \nu_{\mathscr{T}_{2}}(\sigma) U f & =U^{*} \nu_{\mathscr{T}_{2}}(\sigma)\left(\alpha e_{\text {root }_{2}}+\beta U b_{v, n}\right) \\
& =U^{*} \int_{\sigma}\left(\alpha w_{0}(z) e_{\text {root }_{2}}+\beta w_{n+1}(z) U b_{v, n}\right) d A(z) \\
& =\int_{\sigma}\left(\alpha w_{0}(z) e_{\text {root }_{1}}+\beta w_{n+1}(z) b_{v, n}\right) d A(z) \\
& =\nu_{\mathscr{T}_{1}}(\sigma) f
\end{aligned}
$$

which completes the verification of (5.6). One can now define $\tilde{U}: L_{a}^{2}\left(\nu_{\mathscr{T}_{1}}\right) \rightarrow$ $L_{a}^{2}\left(\nu_{\mathscr{T}_{2}}\right)$ by

$$
(\tilde{U} f)(z)=U(f(z))\left(f \in L_{a}^{2}\left(\nu_{\mathscr{T}_{1}}\right), z \in \mathbb{D}\right) .
$$

It is easy to see using (5.3) that $\tilde{U}$ is a unitary map such that $\tilde{U} \mathscr{M}_{z, q}^{(1)}=\mathscr{M}_{z, q}^{(2)} \tilde{U}$. 
Acknowledgment. The authors convey sincere thanks to Gadadhar Misra for some fruitful conversations pertaining to the theory of homogeneous operators. In particular, they acknowledges his help for providing an exact reference for a fact essential in the proof of Proposition 4.4. Further, the last author expresses his gratitude to the Department of Mathematics, IIT Kanpur for their warm hospitality during the preparation of this paper.

\section{REFERENCES}

[1] J. Agler and J. McCarthy, Pick Interpolation and Hilbert Function Spaces, Graduate Studies in Mathematics, 44. American Mathematical Society, Providence, RI, 2002, xx+308 pp.

[2] J. Agler and M. Stankus, m-isometric transformations of Hilbert spaces. I, Integr. Eqn. Op. Th. 21 (1995), 383-429.

[3] D. Alpay and H. Turgay Kaptanoğlu, Toeplitz operators on Arveson and Dirichlet spaces, Integr. Eqn. Op. Th. 58 (2007), 1-33.

[4] D. Alpay and D. Volok, Point evaluation and Hardy space on a homogeneous tree, Integr. Eqn. Op. Th. 53 (2005), 1-22.

[5] A. Anand and S. Chavan, A Moment Problem and Joint q-Isometry Tuples, Complex Anal. Oper. Theory (2015). doi:10.1007/s11785-015-0516-1.

[6] A. Anand, S. Chavan, Z. Jabłoński, and J. Stochel, Subnormality of the Cauchy Dual of a 2-isometry, preprint. arXiv:1702.01264 1 [math.FA]

[7] N. Arcozzi, R. Rochberg, E. Sawyer, and B. Wick, The Dirichlet space: a survey, New York J. Math. 17 (2011), 45-86.

[8] A. Athavale, Some operator-theoretic calculus for positive definite kernels, Proc. Amer. Math. Soc, 112 (1991), 701-708.

[9] B. Bagchi and G. Misra, The homogeneous shifts, J. Funct. Anal. 204 (2003), 293-319.

[10] C. Berg, J. P. R. Christensen, and P. Ressel, Harmonic Analysis on Semigroups, SpringerVerlag, Berlin 1984.

[11] I. Biswas and G. Misra, $S \widetilde{L(2, \mathbb{R})}$-homogeneous vector bundles, Internat. J. Math, 19 (2008), $1-19$.

[12] L. Brown, R. Douglas, P. Fillmore, Unitary equivalence modulo the compact operators and extensions of $C^{*}$-algebras. Proceedings of a conference on operator theory, Dalhousie Univ., Halifax, N.S., pp. 58-128. Lecture Notes in Math., Vol. 345, Springer, Berlin, 1973.

[13] P. Budzynski, P. Dymek, A. Planeta and M. Ptak, Weighted shifts on directed trees. Their multiplier algebras, reflexivity and decompositions, preprint. arXiv:1702.00765 1 [math.FA]

[14] P. Budzynski, P. Dymek and M. Ptak, Analytic structure of weighted shifts on directed trees, Math. Nachr. 2016. DOI: 10.1002/mana.201500448

[15] S. Chavan, D. Pradhan and S. Trivedi, Multishifts on Directed Cartesian Product of Rooted Directed Trees, preprint. arXiv:1607.03860 3 [math.FA]

[16] S. Chavan and R. Kumari, $\mathcal{U}$-invariant Kernels, Defect Operators, and Graded Submodules, New York Jour of Math, 22 (2016), 677-709.

[17] S. Chavan and S. Trivedi, An Analytic Model for Left-Invertible Weighted Shifts on Directed Trees, J. London Math. Soc. 94 (2016), 253-279.

[18] J. Conway, The Theory of Subnormal Operators, Math. Surveys Monographs, 36, Amer. Math. Soc. Providence, RI 1991.

[19] M. Cowen and R. Douglas, Complex geometry and operator theory, Acta Math. 141 (1978), 187-261.

[20] O. El-Fallah, K. Kellay, J. Mashreghi, and T. Ransford, A Primer on the Dirichlet Space, Cambridge Tracts in Mathematics, 203. Cambridge University Press, Cambridge, 2014. xiv +211 pp.

[21] H. Hedenmalm, B. Korenblum, K. Zhu, Theory of Bergman Spaces, Graduate Texts in Mathematics, 199. Springer-Verlag, New York, 2000. x+286 pp.

[22] Z. Jabloński, Hyperexpansive composition operators, Math. Proc. Cambridge Philos. Soc. 135 (2003), 513-526.

[23] Z. Jabłoński, Il Bong Jung, and J. Stochel, Weighted shifts on directed trees, Mem. Amer. Math. Soc. 216 (2012), no. 1017, viii+106.

[24] A. Koranyi and G. Misra, A classification of homogeneous operators in the Cowen-Douglas class, Adv. Math, 226 (2011) 5338-5360.

[25] A. Olofsson, A von Neumann-Wold decomposition of two-isometries, Acta Sci. Math. (Szeged) $\mathbf{7 0}$ (2004), 715-726.

[26] S. Richter, Invariant subspaces of the Dirichlet shift, J. Reine Angew. Math. 386 (1988), 205-220. 
[27] J. Shapiro, Composition Operators and Classical Function Theory, Universitext: Tracts in Mathematics. Springer-Verlag, New York, 1993. xvi+223 pp.

[28] S. Shimorin, Wold-type decompositions and wandering subspaces for operators close to isometries, J. Reine Angew. Math. 531 (2001), 147-189.

Department of Mathematics and Statistics, Indian Institute of Technology Kanpur, INDIA

E-mail address: chavan@iitk.ac.in

E-mail address: dpradhan@iitk.ac.in

School of Mathematics, Harish-Chandra Research Institute, HBNi, Chhatnag Road, Jhunsi, Allahabad 211019, India

E-mail address: shaileshtrivedi@hri.res.in 